\title{
PACIENTES EM HEMODIÁLISE COM DIAGNÓSTICO DE ENFERMAGEM VOLUME DE LÍQUIDOS EXCESSIVO: ASPECTOS SOCIOECONÔMICOS E CLÍNICOS*
}

Maria Isabel da Conceição Dias Fernandes Cavalcanti', Priscila Kaline de Andrade Silva², Anna Lívia de Medeiros Dantas ${ }^{3}$, Maria das Graças Mariano Nunes de Paiva ${ }^{4}$, Marilia Gabriela de Azevedo Araújo ${ }^{5}$, Ana Luisa Brandão de Carvalho Lira ${ }^{6}$

${ }^{1}$ Enfermeira. Mestre em Enfermagem. Universidade Federal do Rio Grande do Norte. Natal-RN-Brasil.

${ }^{2}$ Discente de Enfermagem. Universidade Federal do Rio Grande do Norte. Natal-RN-Brasil.

${ }^{3}$ Enfermeira. Mestre em enfermagem. Universidade Federal do Rio Grande do Norte. Natal-RN-Brasil.

${ }^{4}$ Enfermeira. Universidade Federal do Rio Grande do Norte. Natal-RN-Brasil.

${ }^{5}$ Enfermeira. Universidade Federal do Rio Grande do Norte. Natal-RN-Brasil.

${ }^{6}$ Doutora em Enfermagem. Docente do Curso de Enfermagem da Universidade Federal do Rio Grande do Norte. Natal-RN-Brasil.

RESUMO: Objetivou-se descrever aspectos socioeconômicos e clínicos de pacientes renais crônicos em hemodiálise com o diagnóstico de enfermagem Volume de líquidos excessivo. Estudo transversal, realizado em hospital universitário e clínica de hemodiálise do Nordeste do Brasil, com amostra de 100 pacientes. A coleta de dados em formulário abrangeu os dados clínicos e socioeconômicos, entre dezembro de 2012 a abril de 2013. A maioria dos pacientes com diagnóstico era do sexo feminino, raça parda, aposentado, possúam companheiro, professavam religião, tinham média de idade de 50,4 anos, 6,5 anos de estudo e dois salários mínimos. Ainda, apresentava hipertensão, azotemia e hipercalemia, com mediana de 37 meses com doença renal e 33,5 meses em hemodiálise. Em terapia medicamentosa com cloridrato de sevelamer, ácido fólico, eritropoietina humana recombinante e anti-hipertensivos. Identificar aspectos socioeconômicos e clínicos contribuiu para o conhecimento da equipe de enfermagem acerca do contexto em que os pacientes renais crônicos estão inseridos. DESCRITORES: Fatores socioeconômicos; Diálise renal; Líquidos corporais; Diagnóstico de enfermagem.

\section{PATIENTS RECEIVING HEMODIALYSIS WITH THE NURSINGDIAGNOSISOFFLUIDVOLUMEEXCESS: SOCIOECONOMIC AND CLINICAL ASPECTS \\ PACIENTES EN HEMODIÁLISIS CON DIAGNÓSTICO DEENFERMERÍAVOLUMENDELÍQUIDOSEXCESIVO: ASPECTOS SOCIOECONÓMICOS Y CLÍNICOS}

RESUMEN: El objetivo del estudio fue describir aspectos socioeconómicos y clínicos de pacientes renales crónicos en hemodiálisis con el diagnóstico de enfermería Volumen de líquidos excesivo. Estudio transversal, realizado en hospital universitario y clínica de hemodiálisis del Nordeste de Brasil, con muestra de 100 pacientes. Los datos fueron obtenidos por formulario que abarcó los datos clínicos y socioeconómicos, entre diciembre de 2012 y abril de 2013. La mayoria de los pacientes con el diagnóstico era del sexo femenino, raza parda, jubilado, tenía compañero, profesaba religión, tenía media de edad de 50,4 años, 6,5 años de estudio y dos salarios mínimos. También, presentaban hipertensión, azotemia y hipercalemia, con mediana de 37 meses con enfermedad renal y 33,5 meses en hemodiálisis. En terapia de medicamentos con clorhidrato de sevelamer, ácido fólico, eritropoyetina humana recombinante y anti-hipertensivos. Identificar aspectos socioeconómicos y clínicos contribuye para el conocimiento del equipo de enfermería acerca del contexto en que los pacientes renales crónicos están injeridos. DESCRIPTORES: Factores socioeconómicos; Diálisis renal; Líquidos corporales; Diagnóstico de enfermería.

\footnotetext{
*Artigo extraído da dissertação de mestrado intitulada: Acurácia dos indicadores clínicos do diagnóstico de enfermagem volume de líquidos excessivo em pacientes submetidos à hemodiálise. Universidade Federal do Rio Grande do Norte, 2014.
}

\section{Autor Correspondente:}

Maria das Graças Mariano Nunes de Paiva. Universidade Federal do Rio Grande do Norte. BR 101, s/no - 59072-970 - Natal-RN-Brasil.

E-mail: gracinhamariano@hotmail.com
Recebido: $29 / 08 / 2014$ Finalizado: 13/02/2015 


\section{INTRODUÇÃO}

No ano de 2013, no Brasil, 100.397 pessoas foram submetidas a algum tipo de tratamento dialítico. Dessas, $62,3 \%$ estavam na faixa etária entre 19 e 64 anos e 58\% eram pertencentes ao sexo masculino. Ao analisar o número de pessoas em tratamento dialítico, entre 2000 e 2013, percebe-se um aumento significativo de pacientes diagnosticados com Doença Renal Crônica (DRC) ${ }^{(1)}$.

Na DRC ocorre a perda gradual e irreversível das funções dos rins, repercutindo, em alguns pacientes, na retenção de sódio e água, aumentando, consequentemente, o risco de desenvolvimento de edema, hipertensão arterial e insuficiência cardíaca, sendo necessário, portanto, o início da terapia de substituição renal ${ }^{(2)}$.

Os principais tipos de terapia de substituição renal são: transplante renal, hemodiálise e diálise peritoneal. A hemodiálise destaca-se como o principal tipo de tratamento, a qual consiste na filtração do sangue por uma máquina, cujo objetivo é extrair substâncias tóxicas do sangue e remover o excesso de água. A maioria dos pacientes a realiza três vezes na semana, com duração média de três a quatro horas(2).

Nesse contexto, o paciente renal crônico submetido à hemodiálise apresenta diferentes necessidades e problemas, os quais podem ser identificados pelo enfermeiro por meio de diagnósticos de enfermagem (DE). Dentre os possíveis DE nessa clientela, destaca-se o Volume de líquidos excessivo (VLE), tendo em vista que a retenção de água e eletrólitos é uma das características da falência renal. Além disso, o paciente apresenta outros sinais e sintomas inseridos nesse diagnóstico como anasarca, azotemia, congestão pulmonar, dispneia, edema, oligúria, ortopneia e ruídos respiratórios adventícios ${ }^{(3)}$.

Estudos sobre o perfil de diagnósticos de enfermagem nos pacientes com DRC mostraram que mais de $50 \%$ desses pacientes possui $\mathrm{VLE}^{(3-5)}$. Em virtude da elevada frequência de tal diagnóstico nessa clientela, compreendeu-se como importante caracterizar o perfil socioeconômico e clínico de pacientes submetidos à hemodiálise com esse $\mathrm{DE}$, uma vez que irá direcionar ações de enfermagem em clientela semelhante, proporcionando cuidado mais eficaz e qualificado.
Estudos sobre o perfil socioeconômico auxiliam os profissionais a estabelecerem um plano de cuidados direcionado às relações existentes entre ambos. Observou-se em estudo que o VLE estava mais susceptível em pacientes idosos, do sexo feminino, profissionalmente inativos, com sobrepeso/obesidade ou com maior número de condições clínicas ${ }^{(4)}$.

Diante do exposto, cabe aos enfermeiros considerarem o contexto no qual os indivíduos com DRC estão inseridos e não somente seus aspectos fisiopatológicos. Logo, surgem as questões norteadoras deste estudo, a saber: qual o perfil socioeconômico dos pacientes em hemodiálise com o diagnóstico de enfermagem VLE? Qual o perfil clínico dos pacientes em hemodiálise com o diagnóstico de enfermagem VLE?

Dessa forma, o estudo apresenta como objetivo descrever os aspectos socioeconômicos e clínicos dos pacientes renais crônicos em hemodiálise com o diagnóstico de enfermagem VLE. Assim, a partir deste estudo os profissionais envolvidos no cuidar de pacientes com DRC e com diagnóstico de enfermagem VLE poderão compreender mais sobre os diferentes perfis de pacientes e obter maior subsídio para a elaboração de um plano de cuidados direcionado, com vistas a proporcionar assistência de qualidade, considerando a realidade e necessidades de cada paciente.

\section{MÉTODO}

Trata-se de um estudo transversal, com abordagem quantitativa, realizado no setor de hemodiálise de um hospital universitário e de uma clínica de hemodiálise no Nordeste do Brasil. A coleta conjunta nessas duas instituições proporcionou um quantitativo de pacientes adequado para a pesquisa, no tempo determinado.

A população foi de 300 indivíduos, calculados por meio da média mensal de pacientes das clínicas referidas. A amostra foi calculada considerandose o nível de confiança de $95 \%$, a sensibilidade de $85 \%$; a metade do comprimento dos intervalos de confiança de $10 \%$ e proporção de indivíduos, supostamente, com o diagnóstico em estudo de 50. Logo, obteve-se um total de 98 indivíduos, porém optou-se por finalizar em 100 pacientes.

Como critérios de inclusão, estipularam-se: ser portador de IRC; realizar tratamento hemodialítico; 
estar na primeira hora de hemodiálise no momento da coleta; ter 18 ou mais anos de idade. Já como critérios de exclusão: estar prejudicado físico e mentalmente de forma que não fosse possível realizar a coleta de dados.

Foi utilizado um instrumento do tipo formulário, com perguntas abrangendo os dados clínicos e socioeconômicos, contemplando as seguintes variáveis: hipertensão arterial sistêmica, diabetes mellitus, exames laboratoriais, medicamentos prescritos, sinais vitais, tempo de doença renal, tratamento hemodialítico, sexo, raça, estado civil, religião, ocupação, idade, anos de estudo e renda familiar. A coleta ocorreu entre os meses de dezembro de 2012 a abril de 2013. Os participantes que atendiam ao critério de inclusão foram convidados a participar da pesquisa. Após o consentimento foi realizada a assinatura do termo de consentimento livre e esclarecido. Em seguida os pacientes foram submetidos à entrevista e ao exame físico.

Os dados referentes à prevalência do diagnóstico de enfermagem estudado e os aspectos socioeconômicos foram analisados com o auxílio do IBM SPSS Statistic versão 19.0 for Windows. Para a análise dos dados socioeconômicos foi utilizada a estatística descritiva, sendo calculadas as frequências relativa e absoluta, a média, a mediana, o mínimo, o máximo e o desvio padrão, e sendo aplicado o teste de Kolmogorov-Smirnov para verificação da normalidade dos dados $(p<0,05)$.

Esse estudo foi apreciado e aprovado pelo Comitê de Ética em Pesquisa sob o Parecer $n^{\circ}$ 148.428 e Certificado de Apresentação para Apreciação Ética (CAAE) nº 08696212.7.0000.5537.

\section{RESULTADOS}

$\mathrm{Na}$ amostra do presente estudo, composta por 100 pacientes submetidos à hemodiálise, o diagnóstico de enfermagem VLE esteve presente em 82 pacientes. Os resultados serão expostos em três tabelas que irão exibir os dados socioeconômicos, dados clínicos e medicamentos utilizados por esses pacientes.

A partir dos dados expostos observa-se que dos 82 pacientes com o diagnóstico de enfermagem investigado, a maioria era do sexo feminino. Grande parte da amostra era parda,
Tabela 1 - Caracterização socioeconômica dos pacientes submetidos à hemodiálise que apresentavam o diagnóstico de enfermagem Volume de líquidos excessivo. Natal, RN, 2014

\begin{tabular}{|c|c|c|c|}
\hline \multicolumn{2}{|l|}{ Variáveis } & $\mathbf{N}$ & $\%$ \\
\hline \multicolumn{4}{|l|}{ Sexo } \\
\hline \multicolumn{2}{|c|}{ Feminino } & 44 & 53,7 \\
\hline \multicolumn{2}{|c|}{ Masculino } & 38 & 46,3 \\
\hline \multicolumn{4}{|l|}{ Raça } \\
\hline \multicolumn{2}{|l|}{ Pardo } & 43 & 52,4 \\
\hline \multicolumn{2}{|l|}{ Negro } & 21 & 25,6 \\
\hline \multicolumn{2}{|c|}{ Branco } & 17 & 20,8 \\
\hline \multicolumn{2}{|c|}{ Amarelo } & 01 & 1,2 \\
\hline \multicolumn{4}{|c|}{ Estado civil } \\
\hline \multicolumn{2}{|c|}{$\begin{array}{c}\text { Com } \\
\text { companheiro }\end{array}$} & 46 & 56,1 \\
\hline \multicolumn{2}{|c|}{$\begin{array}{c}\text { Sem } \\
\text { companheiro }\end{array}$} & 36 & 43,9 \\
\hline \multicolumn{4}{|l|}{ Religião } \\
\hline \multicolumn{2}{|c|}{ Católico } & 55 & 67,1 \\
\hline \multicolumn{2}{|c|}{ Evangélico } & 16 & 19,5 \\
\hline \multicolumn{2}{|c|}{ Sem religião } & 05 & 6,1 \\
\hline \multicolumn{2}{|l|}{ Espírita } & 04 & 4,9 \\
\hline \multicolumn{2}{|c|}{ Candomblé } & 01 & 1,2 \\
\hline \multicolumn{2}{|c|}{ Catequista } & 01 & 1,2 \\
\hline \multicolumn{4}{|c|}{ Ocupação } \\
\hline \multicolumn{2}{|c|}{ Aposentado } & 45 & 54,9 \\
\hline \multicolumn{2}{|c|}{ Beneficiado } & 22 & 26,8 \\
\hline \multicolumn{2}{|c|}{ Nenhum } & 09 & 11,0 \\
\hline \multicolumn{2}{|l|}{ Ativo } & 04 & 4,9 \\
\hline \multicolumn{2}{|c|}{ Pensionista } & 02 & 2,4 \\
\hline & Idade & Anos de estudo & Renda familiar \\
\hline Média & 50,4 & 8,1 & 2,8 \\
\hline $\begin{array}{l}\text { Desvio } \\
\text { padrão }\end{array}$ & 16,2 & 5,0 & 2,6 \\
\hline Mediana & 51,5 & 6,5 & 2,0 \\
\hline Mínimo & 19,0 & 0,0 & 0,0 \\
\hline Máximo & 86,0 & 18,0 & 15,0 \\
\hline Valor $p$ & 0,2 & 0,0 & 0,0 \\
\hline
\end{tabular}

* Teste de Kolmogorov-Smirnov $(\mathrm{p}<0,05) ;{ }^{* *}$ Idade em anos; ***Renda em salários mínimos.

possuía companheiro, professava algum tipo de religião, sendo a maioria católica. Em relação à ocupação, mais da metade da clientela recebia algum tipo de benefício e residia na capital.

Com relação à idade, obteve-se média de 50,4 anos $( \pm 16,2)$, com mínimo de 19 e máximo de 86 anos. Em relação às variáveis anos de estudo e 
renda, essas apresentaram variação assimétrica (valor $\mathrm{p}<0,05$ ), indicando que metade da amostra tinha até 6,5 anos de estudo e renda de dois salários mínimos, correspondendo a $\mathrm{R} \$ 1356,00$ no momento da pesquisa.

A partir dos dados expostos, observa-se que dos 82 pacientes com o DE Volume de líquidos excessivo, a maior parte apresentava hipertensão. Quanto aos exames laboratoriais, 100\% dos pacientes tinham alteração da ureia e creatinina, a maioria possuía valores normais de sódio e cálcio, enquanto mais da metade tinha alteração de potássio e diminuição de hemoglobina e hematócrito.

Em relação às variáveis meses de doença renal, meses de tratamento hemodialítico, pressão arterial diastólica, frequência respiratória e pulso, essas apresentaram variação assimétrica, assim,

Tabela 2 - Dados clínicos dos pacientes submetidos à hemodiálise que apresentavam o diagnóstico de enfermagem Volume de líquidos excessivo. Natal, RN, 2014

\begin{tabular}{|c|c|c|c|c|c|c|}
\hline \multirow{2}{*}{$\begin{array}{l}\text { Variáveis } \\
\text { Hipertensão }\end{array}$} & \multicolumn{3}{|c|}{$\mathbf{N}$} & \multicolumn{3}{|c|}{$\%$} \\
\hline & & & & & & \\
\hline \multicolumn{2}{|l|}{ Sim } & \multicolumn{3}{|l|}{66} & \multicolumn{2}{|l|}{80,5} \\
\hline Não & \multicolumn{3}{|c|}{16} & \multicolumn{3}{|c|}{19,5} \\
\hline \multicolumn{7}{|l|}{ Diabetes } \\
\hline Sim & \multicolumn{3}{|c|}{24} & \multicolumn{3}{|c|}{29,3} \\
\hline Não & \multicolumn{3}{|c|}{58} & \multicolumn{3}{|c|}{70,7} \\
\hline \multicolumn{7}{|l|}{ Ureia } \\
\hline Normal & \multicolumn{3}{|c|}{-} & \multicolumn{3}{|c|}{-} \\
\hline Alterado & \multicolumn{3}{|c|}{82} & \multicolumn{3}{|c|}{100} \\
\hline \multicolumn{7}{|l|}{ Creatinina } \\
\hline Normal & \multicolumn{3}{|c|}{-} & \multicolumn{3}{|c|}{-} \\
\hline Alterado & \multicolumn{3}{|c|}{82} & \multicolumn{3}{|c|}{100} \\
\hline \multicolumn{7}{|l|}{ Sódio } \\
\hline Normal & \multicolumn{3}{|c|}{48} & \multicolumn{3}{|c|}{58,5} \\
\hline Alterado & \multicolumn{3}{|c|}{34} & \multicolumn{3}{|c|}{41,5} \\
\hline Cálcio & & & & & & \\
\hline Normal & & 47 & & & 57,3 & \\
\hline Alterado & & 35 & & & 42,7 & \\
\hline Potássio & & & & & & \\
\hline Normal & & 22 & & & 26,8 & \\
\hline Alterado & & 60 & & & 73,2 & \\
\hline Hemoglobina & & & & & & \\
\hline Normal & & 22 & & & 26,8 & \\
\hline Diminuído & & 60 & & & 73,2 & \\
\hline Hematócrito & & & & & & \\
\hline Normal & & 04 & & & 4,9 & \\
\hline Diminuído & & 78 & & & 95,1 & \\
\hline & Média & $\begin{array}{l}\text { Desvio } \\
\text { Padrão }\end{array}$ & Mediana & Mínimo & Máximo & Valor $\mathbf{p}^{*}$ \\
\hline Meses de doença renal & 67,5 & 63,4 & 37,0 & 1,0 & 264,0 & 0,0 \\
\hline Meses de tratamento hemodialítico & 51,9 & 55,5 & 33,5 & 1,0 & 216,0 & 0,0 \\
\hline Pressão arterial sistólica & 137,1 & 25,5 & 140,0 & 80,0 & 220,0 & 0,3 \\
\hline Pressão arterial diastólica & 87,0 & 15,9 & 85,0 & 60,0 & 140,0 & 0,0 \\
\hline Frequência respiratória & 20,3 & 4,1 & 20,0 & 11,0 & 31,0 & 0,0 \\
\hline Pulso & 78,2 & 15,1 & 73,5 & 54,0 & 121,0 & 0,0 \\
\hline
\end{tabular}


obteve-se mediana de 37 meses, 33,5 meses, 85,0 milímetros de mercúrio ( $\mathrm{mmHg}$ ), 20 movimentos respiratórios por minuto e 73,5 batimentos cardíacos por minuto, respectivamente. Já a pressão arterial sistólica apresentou média de $137,1 \mathrm{mmHg}( \pm 25,5)$, com máximo de 220,0 e mínimo de 80,0.

Tabela 3 - Medicamentos utilizados pelos pacientes com o diagnóstico de enfermagem Volume de líquidos excessivo. Natal, RN, 2014

\begin{tabular}{lcc}
\hline Variáveis & $\mathbf{N}$ & $\mathbf{\%}$ \\
\hline $\begin{array}{l}\text { Cloridrato de } \\
\text { sevelamer }\end{array}$ & 69 & 84,1 \\
\hline Ácido fólico & 64 & 78,0 \\
\hline $\begin{array}{l}\text { Eritropoetina } \\
\text { humana } \\
\text { recombinante }\end{array}$ & 62 & 75,6 \\
\hline Anti-hipertensivo & 62 & 75,6 \\
\hline Cianocobalamina & 51 & 62,2 \\
\hline Ferripolimaltose & 36 & 43,9 \\
\hline $\begin{array}{l}\text { Carbonato de } \\
\text { cálcio }\end{array}$ & 33 & 40,2 \\
\hline $\begin{array}{l}\text { Analgésico e } \\
\text { antipirético }\end{array}$ & 11 & 13,4 \\
\hline Insulina & 9 & 11,0 \\
\hline Omeprazol & 3 & 3,7 \\
\hline Pantoprazol & 3 & 3,7 \\
\hline Sinvastatina & 3 & 3,7 \\
\hline Clonazepam & 2 & 2,4 \\
\hline Antiarrítmico & 1 & 1,2 \\
\hline $\begin{array}{l}\text { Cloridrato de } \\
\text { fluoxetina }\end{array}$ & 1 & 1,2 \\
\hline Calcitriol & 1 & 1,2 \\
\hline Alopurinol & 1 & 1,2 \\
\hline Prednisona & 1 & 1,2 \\
\hline & & \\
\hline
\end{tabular}

A partir dos dados apresentados na tabela acima, observou-se que os medicamentos mais utilizados pelos pacientes com o DE Volume de líquidos excessivo foram: carbonato de sevelamer, ácido fólico, eritropoietina humana recombinante, anti-hipertensivo, cianocobalamina, ferripolimaltose, carbonato de cálcio, analgésico e antipirético e insulina.

\section{DISCUSSÃO}

Com relação à amostra da pesquisa (100 pacientes), observou-se que $82 \%$ obtiveram o diagnóstico de enfermagem VLE, caracterizando mais da metade dos indivíduos. Dado corroborado por estudos que também evidenciam a presença desse diagnóstico em pacientes submetidos à hemodiálise $^{(3,5-6)}$.

A alta porcentagem desse diagnóstico pode ser explicada fisiologicamente, uma vez que indivíduos com DRC apresentam redução na taxa de filtração glomerular, ocasionando redução na excreção renal de água e eletrólitos que se acumulam e desencadeiam o acúmulo de líquido(7).

Verificou-se que dos 82 pacientes, a maioria era do sexo feminino fato que contrapõe às pesquisas encontradas na literatura cujos dados mostram que o percentual de pacientes em hemodiálise, do sexo masculino, é maior ${ }^{(3,8)}$. Nesse aspecto, estudo aponta que as mulheres submetidas à hemodiálise apresentam escores menores na qualidade de vida e apresentam um maior risco de morte quando comparadas aos homens. Ademais, atrelado a isso, o sexo feminino mantêm a função de fornecer cuidado ao lar e aos filhos, realidade que pode ser a responsável por aumentar o estresse físico e mental( ${ }^{(9)}$. Destarte, supõe-se que essa diminuição na qualidade de vida possa estar associada ao maior contingente de mulheres com VLE.

Com relação à raça, mais da metade foram classificados como pardos, diferente de alguns estudos que indicam a raça branca como a mais recorrente ${ }^{(3,10-12)}$. Enquanto outro estudo considera a raça negra e mulata com maior risco para a doença renal terminal ${ }^{(12)}$.

Sobre estado civil mais da metade dos pacientes relataram ter companheiros o que foi evidenciado em outros estudos ${ }^{(11-13)}$. A presença da família é fundamental ao paciente renal em hemodiálise, em virtude de essa auxiliar no enfrentamento da doença, bem como na adesão ao tratamento ${ }^{(14)}$.

Concernente à presença de religiosidade, a qual esteve presente em grande parte dos entrevistados, também desempenha um ponto importante, tendo-se em vista que contribui para o processo de reabilitação dos indivíduos 
submetidos à diálise. Para essas pessoas, acreditar em um ser superior configura-se em um suporte, o qual os ajuda a enfrentar as adversidades decorrentes da doença e do tratamento. Além disso, representa também a esperança de cura ${ }^{(14)}$.

Referente à ocupação e a renda, a maioria dos participantes era de aposentados e renda mensal de dois salários mínimos. O número elevado de aposentados pode ser justificado devido ao benefício adquirido pelo Instituto Nacional de Seguridade Social (INSS), após constatação da doença renal crônica ${ }^{(11)}$. Destacando-se que a minoria dos indivíduos possuía algum vínculo empregatício, realidade decorrente da limitação imposta aos pacientes submetidos à hemodiálise, pois sua realização demanda tempo, além disso, há os problemas físicos acarretados pela insuficiência renal, tais como: náuseas, malestar, fadiga e perda das forças, dificultando a realização do trabalho ${ }^{(15)}$.

A renda de dois salários para a família do paciente relaciona-se com o perfil das instituições pesquisadas, as quais atendem pacientes cujo plano de saúde é o Sistema Único de Saúde (SUS), sendo este o principal responsável pelo pagamento dos tratamentos dialíticos no Brasil $^{(1)}$. Logo, indivíduos com baixo nível econômico realizam o tratamento por meio do SUS, visto que estes não possuem condições favoráveis para pagar planos de saúde privados, corroborando, com outros autores, em um estudo de perfil com doentes renais crônicos, cujos indivíduos com baixo nível econômico são usuários do SUS ${ }^{(16)}$.

Nesse sentido, a baixa condição socioeconômica, por sua vez, interfere na qualidade da adesão ao tratamento, principalmente no que se refere à alimentação, além da dificuldade de acesso ao serviço de saúde, ao transporte, ao tratamento farmacológico e dialítico, favorecendo uma sobrevida indigna ${ }^{(13)}$. Nesse sentido, acredita-se que esse perfil tenha favorecido a presença do VLE na amostra aqui estudada.

Com relação à idade, os resultados foram semelhantes aos dados do Censo de Nefrologia ${ }^{(1)}$. Nessa perspectiva, estudo assevera que indivíduos em hemodiálise com maior faixa etária possuem menor taxa de sobrevida ${ }^{(9,17)}$. Assim, tendo em vista que neste estudo grande parte da amostra foi enquadrada em uma faixa de idade avançada e considerando a presença frequente do VLE, que pode precipitar alguns problemas de saúde nesses indivíduos, acredita-se que a taxa de sobrevida nos pacientes investigados tende a diminuir, em virtude desses dois fatores.

Referente à escolaridade, a mediana de estudo foi de 6,5 anos. Dado similar com a literatura, a qual revela pacientes submetidos ao tratamento hemodialítico com o ensino fundamental incompleto, interferindo na compreensão dos indivíduos acerca da sua doença, sendo a educação de fundamental importância para a adesão ao tratamento. Logo, cabe ao enfermeiro adequar às orientações fornecidas a essa clientela a fim de proporcionar maior entendimento sobre o tratamento e as restrições necessárias ${ }^{(11-13)}$.

As comorbidades apresentadas pelos pacientes pesquisados observou-se que grande parte possuía hipertensão arterial sistêmica (HAS), resultado similar a outras pesquisas nas quais a HAS está associada a alterações funcionais e/ou estruturais de órgãos-alvo, como os rins ${ }^{(1,13,18-19)}$. Estudos asseveram que a elevada taxa de doenças associadas como diabetes melitus e hipertensão arterial contribuem para a alta prevalência de doenças cardiovasculares (DCV) nos pacientes com DRC, uma vez que causam lesão no tecido renal e no sistema cardiovascular ${ }^{(20)}$.

Nesse sentido, embora identificada com menor porcentagem neste estudo, o diabetes, se instala de forma gradativa e assintomática, evoluindo com a perda da função renal e maior necessidade de diálise, limitando a qualidade de vida e aumentando o risco de morte. Uma das principais complicações é a nefropatia diabética, resultante da longa exposição à hiperglicemia, mau controle da hipertensão, dos níveis do colesterol, do hábito de fumar e também de fatores genéticos. Sua prevenção é muito importante para evitar e/ou reduzir a lesão renal, portanto é essencial realizar o controle glomerular ${ }^{(21-22)}$.

Destarte, tendo em vista a frequência elevada da HAS e do diabetes em pessoas com DRC, a redução do risco de mortalidade dos indivíduos com DRC deverá ser um papel importante do enfermeiro, o qual deve orientar o paciente a respeito da importância do cumprimento das práticas protetoras à saúde, como a mudança no estilo de vida, interrupção do consumo de tabaco, redução da ingesta de sódio e proteínas, diminuição do peso, e prática de atividade física 
pelo menos 30 minutos, cinco vezes por semana, além do tratamento farmacológico adequado ${ }^{(23-24)}$.

Referente aos sinais vitais avaliados no início da coleta observou-se que a frequência respiratória foi considerada normal dentro dos parâmetros encontrados na literatura entre $16 \mathrm{e}$ 20 mrpm. O pulso também se encontra dentro da normalidade cujos valores são de 60 a 100 bpm $^{(25)}$.

Por fim, de acordo com a classificação estipulada pela literatura, a pressão arterial sistólica e diastólica apresentou-se limítrofe, a sistólica entre 130-139 mmHg e a diastólica entre 85-89 mmHg. A sua mensuração foi por meio do método indireto, com tensiômetro aneroide. Segundo a classificação, considera-se hipertensão arterial quando a pressão sistólica for igual ou maior que 140 mmHg e ou pressão diastólica maior ou igual a $90 \mathrm{mmHg}^{(24)}$.

Referente aos exames laboratoriais houve alteração nos valores de ureia e creatinina em $100 \%$ da amostra. Os rins eliminam os produtos de degradação do metabolismo do corpo, sendo a ureia o principal produto do metabolismo da proteína e a creatinina o produto da degradação endógena dos músculos esqueléticos. Entretanto, no paciente com DRC, à medida que a taxa de filtração glomerular (TFG) diminui, a excreção renal dessas substâncias reduz na mesma proporção $^{(2)}$, as quais ficam acumuladas no sangue, fato que explica a alteração nos 82 pacientes.

Avaliaram-se, ainda, as alterações eletrolíticas, sendo observado, principalmente, nos níveis séricos de potássio. A hiperpotassemia é comumente encontrada em pacientes com insuficiência renal aguda e crônica com uma taxa de filtração glomerular menor que 10 a $20 \%$ do normal. Esse excesso tem efeito no miocárdio e quando grave causa fraqueza do músculo esquelético e paralisia, relacionada com o bloqueio da despolarização muscular. Além disso, podem surgir manifestações como náuseas, cólica intestinal e diarreia ${ }^{(2)}$.

A diminuição da hemoglobina e do hematócrito em grande parte dos pacientes, reflete a redução da função renal na DRC, causando um quadro de anemia. Isso ocorre devido à ineficiência na produção da eritropoietina, hormônio produzido, principalmente, pelos rins que estimula a medula óssea a produzir eritrócitos. Como consequência surgem sintomas como fadiga e falta de $\operatorname{ar}^{(2,26-27)}$.
Com relação aos meses de doença renal e meses de tratamento hemodialítico, obtiveram-se medianas de 37 e 33,5 meses, respectivamente. Dado semelhante foi encontrado no censo 2013 da Sociedade Brasileira de Nefrologia ${ }^{(1)}$. Vale salientar que a doença renal contém cinco estágios e a diálise apenas é indicada quando o último estágio é atingido, no qual a taxa de filtração glomerular está menor que $15 \mathrm{l} / \mathrm{mim}^{(24)}$. Portanto, o tempo de doença renal nem sempre corresponde ao tempo de tratamento dialítico.

Ressalta-se a importância no controle da pressão, uma vez que grande parte dos renais crônicos apresenta hipertensão arterial sistêmica. Essa quando não controlada tende a causar maior dano ao sistema renal, contribuindo para uma piora do quadro, portanto para uma maior eficácia no controle, o tratamento deve ser individualizado, considerando a causa da DRC e a presença de DCV pré-existente ${ }^{(19)}$.

Como identificado neste estudo, a DRC origina diversas complicações, dentre essas a hiperfosfatemia que consiste no aumento da concentração plasmática de fosfato. Essa quando associada ao cálcio pode provocar à calcificação das artérias coronárias, e consequente doença cardíaca isquêmica, infarto agudo do miocárdio, parada cardíaca e morte súbita. Logo, para o seu tratamento se faz necessário o uso de medicamentos como o Cloridrato de sevelamer que se encontra em primeiro lugar na lista dos fármacos usados por essa população ${ }^{(28)}$.

Os medicamentos utilizados para tratamento da anemia, muito comum nos renais crônicos devido à deficiência de eritropoetina produzida pelos rins, também tiveram grande número de usuários, como o Ácido fólico e o Eritropoetina recombinante humana. Este último, sendo uma das drogas de maior gasto no Brasil ${ }^{(28)}$.

Além desses, os anti-hipertensivos também tiveram grande número de utilização, em virtude de grande parte da amostra ser portador da HAS. Ressalta-se que pacientes com DRC associada à hipertensão têm maior risco de desenvolver doença cardiovascular, dessa forma, a pressão arterial deve ser controlada de forma rigorosa para que esse risco seja reduzido ${ }^{(20,29)}$.

A baixa condição socioeconômica dos pacientes desse estudo associado às dificuldades com alimentação e tratamento farmacológico faz do Sistema Único de Saúde o responsável 
por custear o tratamento de grande parte da população, tornando-se, assim, elevado os gastos do governo. No entanto, se não fossem realizados, os usuários dificilmente teriam condições de arcar com os custos, em virtude da cronicidade da doença, sendo necessário o uso constante desses medicamentos e do tratamento em geral.

Além disso, compreende-se que o sistema financeiro brasileiro e a saúde da população carecem de um melhor investimento em medidas preventivas na atenção primária, para promoção da saúde daqueles indivíduos com fatores de risco para o desenvolvimento da DRC.

\section{CONCLUSÃO}

Neste estudo foram avaliados 100 pacientes com diagnóstico de doença renal crônica e submetidos à hemodiálise, dos quais a maioria possuía o diagnóstico de enfermagem VLE. Desses, grande parte foi de indivíduos do sexo feminino, pardos, com idade média de 51 anos, com companheiros, vínculo religioso, aposentados, com renda de dois salários mínimos e baixa escolaridade.

Ademais, ao analisar as variáveis socioeconômicas e a prevalência do diagnóstico de enfermagem VLE nessa clientela específica, verificou-se que o sexo, o estado civil, a religiosidade, a ocupação, a renda, a escolaridade e a idade são variáveis similares a outros estudos sobre perfil nessa clientela e segundo a literatura se relacionam com a presença deste diagnóstico.

Com relação aos dados clínicos, a maior parte possuía como comorbidades hipertensão e diabetes. Os exames laboratoriais de ureia, creatinina, potássio, hemoglobina e hematócritos apresentaram alterações. No que se refere ao tempo com doença renal observou-se que esse foi ligeiramente maior que o tempo em tratamento hemodialítico. Os medicamentos utilizados são variados, porém prevalecem os destinados ao tratamento das complicações provocadas pela doença renal crônica e hemodiálise, dados também condizentes com estudos similares.

Conclui-se, portanto, que o conhecimento dos aspectos socioeconômicos e clínicos pela equipe de enfermagem é relevante, pois contribui para aumentar a gama de conhecimentos acerca do contexto em que os pacientes renais crônicos estão inseridos, o qual não pode ser dissociado dos cuidados clínicos, sendo imprescindíveis, então, estudos contínuos com essa temática, principalmente por se tratar de uma doença crônica. Ademais, o perfil clínico e socioeconômico favorece a uma assistência eficaz e precisa, e assim, cuidado de enfermagem qualificado e embasado cientificamente.

\section{REFERÊNCIAS}

1. Sociedade Brasileira de Nefrologia [Internet]. Censo de diálise; 2013 [Acesso em 20 maio 2014]. Disponível em: http://www.sbn.org.br/censos

2. Smeltzer SC, Bare BG, Hinkle JL, Cheever KH. Brunner \& Suddarth: Tratado de Enfermagem Médico Cirúrgica. $12^{\mathrm{a}}$ ed. Rio de Janeiro: Guanabara Koogan; 2012.

3. Bisca MN, Marques IR. Perfil de diagnósticos de enfermagem antes de iniciar o tratamento hemodialítico. Rev Bras Enferm. [Internet] 2010; 63(3) [acesso em 13 novembro de 2013]. Disponível em: http://www.scielo.br/scielo.php?script=sci_ arttext\&pid=S0034-71672010000300014

4. Nunciaroni AT, Gallani MCBJ, Agondi RF, Rodrigues RCM, Castro LT. Caracterização dos diagnósticos de enfermagem de pacientes internados em uma unidade de cardiologia. Rev Gaúcha Enferm. [Internet] 2012; 33(1) [acesso em 13 novembro 2013]. Disponível em: http://www.scielo.br/scielo.php?pid=S1983$14472012000100005 \&$ script=sci_arttext

5. Triquez SL, Dallacosta FM. Perfil dos pacientes com insuficiência renal aguda na unidade de terapia intensiva e principais diagnósticos de enfermagem. Unoesc \& Ciência. [Internet] 2012; 3(2) [acesso em 13 novembro 2013]. Disponível em: http://www.scielo.br/scielo. php?pid=S1983-14472012000100005\&script=sci_ arttext

6. Dallé J, Lucena AF. Diagnósticos de enfermagem identificados em pacientes hospitalizados durante sessões de hemodiálise. Acta Paul Enferm. [Internet] 2012; 25(4) [acesso em 14 novembro 2013]. Disponível em: http://www.scielo.br/scielo.php?script=sci_ arttext\&pid=S0103-21002012000400004

7. Guyton AC, Hall JE. Tratado de fisiologia médica. $12^{\mathrm{a}}$ ed. Rio de Janeiro: Elsivier; 2012.

8. Holanda RH, Silva V.M. Diagnósticos de enfermagem de pacientes em tratamento hemodialítico. Rev Rene. [Internet] 2009; 10(2) [acesso em 14 novembro 2013]. Disponível em: http://www.revistarene.ufc.br/10.2/ html/10_2_3.html 
9. Szuster DAC, Caiaffa WT, Andrade EIG, Acurcio FA, Cherchiglia ML. Sobrevida de pacientes em diálise no SUS no Brasil. Cad Saúde Pública. [Internet] 2012; 28(3) [acesso em 2 fevereiro 2014]. Disponível em: http://www.scielo.br/pdf/csp/v28n3/02.pdf

10. Ribeiro RCHM, Ferrari RR, Bertolin DC, Canova GCM, Lima LCEQ, Ribeiro DF. Caracterização e etiologia da insuficiência renal crônica em unidade de nefrologia do interior do estado de São Paulo. Acta Paul Enferm. [Internet] 2008; 21(n.esp) [acesso em 2 fevereiro 2014]. Disponível em: http://www.scielo.br/pdf/ape/v21nspe/ a13v21ns.pdf

11. Vasconcelos CR, Dutra DA, Oliveira EM, Fernandes S. Perfil socioeconômico e clínico de um grupo de diabéticos em tratamento hemodialítico em Curitiba. Revista Uniandrade. [Internet] 2013; 14(2) [acesso em 2 fevereiro 2014]. Disponível em: http:// www.uniandrade.br/revistauniandrade/index.php/ revistauniandrade/article/view/60/56

12. Moraes CE, Cerolli CF, Silva VS, Franco RJS, Habermann F, Matsubara BB, Martin LC. Preditores de insuficiência renal crônica em pacientes de centro de referência em hipertensão arterial. Ver Assoc Med Bras. [Internet] 2009; 55(3) [acesso em 28 janeiro 2015]. Disponível em: http://www.scielo.br/pdf/ramb/ v55n3/v55n3a15.pdf

13. Frazão CMFQ, Ramos VP, Lira ALBC. Qualidade de vida de pacientes submetidos a hemodiálise. Rev Enferm UERJ. [Internet] 2011; 19(4) [acesso em 2 fevereiro]. Disponível em: http://www.facenf.uerj.br/ v19n4/v19n4a12.pdf

14. Schwartz E, Muniz RM, Burille A, Zillmer JGV, Silva DA, Feijó AM et al. As redes de apoio no enfrentamento da doença renal crônica. Revista Mineira de Enferm. [Internet] 2009; 13(2) [acesso em 4 fevereiro 2014]. Disponível em: http://www.reme. org.br/artigo/detalhes/179

15. Coutinho NPS, Vasconcelos GM, Lopes MLH, Wadie WCA, Tavares MCH. Qualidade de vida de pacientes renais crônicos em hemodiálise. Rev Pesq Saúde. [Internet] 2010; 11(1) [acesso em 4 fevereiro 2014]. Disponível em: http://www.periodicoseletronicos. ufma.br/index.php/revistahuufma/article/view/328

16. Martínez BB, Silva FM, Veiga VT, Custódio RP, Silva JV. Desigualdade social em pacientes renais crônicos. Rev Bras Clin Med São Paulo. [Internet] 2011; 9(3) [acesso em 2 fevereiro 2015]. Disponível em: http://files.bvs. br/upload/S/1679-1010/2011/v9n3/a1977.pdf

17. Azevedo DF, Correa MC, Botre L, Mariano RM, Assis RR, Grossi L, et al. Sobrevida e causas de mortalidade em pacientes hemodialíticos. Rev Med Minas Gerais. [Internet] 2009; 19(2) [acesso em 4 fevereiro 2014]. Disponível em: http://www.medicina.ufmg.br/rmmg/ index.php/rmmg/article/viewArticle/111
18. Braga FLM, Arruda IKG, Diniz AS, Cabral PC, Lemos MCC, Braga MDM, et al. Disfunção renal e marcadores inflamatórios em hipertensos atendidos em hospital universitário. Arq Bras Cardiol. [Internet] 2013; 100(6) [acesso em 4 julho 2014]. Disponível em: http://www. scielo.br/pdf/abc/v100n6/aop_5047.pdf

19. Sociedade Brasileira de Cardiologia. VI Diretrizes brasileiras de hipertensão. Rev Bras Hipertens. [Internet] 2010; 17(1) [acesso em 4 julho 2014]. Disponível em: http://departamentos.cardiol.br/dha/ vidiretriz/vidiretriz.asp

20. Ammirati AL, Canziani MEF. Fatores de risco da doença cardiovascular nos pacientes com doença renal crônica. J Bras Nefrol. [Internet] 2009; 31 Suppl 1 [acesso em 5 julho 2014]. Disponível em: http:// www.sbn.org.br/pdf/diretrizes/JBN_educacional_II/9Canziani.pdf

21. Braga SFM, Peixoto SV, Gomes IC, Acúrcio FA, Andrade EIG, Cherchiglia ML. Fatores associados com a qualidade de vida relacionada à saúde de idosos em hemodiálise. Rev Saúde Pública. [Internet] 2011; 45(6) [acesso em 6 julho 2014]. Disponível em: http://www.scielo.br/scielo.php?script=sci_ arttext\&pid=S0034-89102011000600015

22. Sociedade Brasileira de Diabetes [Internet]. Diabetes e doença renal crônica; 2014 [acesso em 7 julho 2014]. Disponível em: http://www.diabetes.org.br/artigossobre-diabetes/59-diabetes-e-doenca-renal-cronica

23. Gansevoort RT, Rotter RC, Hemmelgarn GR, Jafar TH, Heerspink HJL, Mann JF, et al. Chronic kidney disease and cardiovascular risk: epidemiology, mechanisms, and prevention. Lancet. [Internet] 2013; (382) [acesso em 7 julho 2014]. Disponível em: http:// www.thelancet.com/journals/lancet/article/PIIS01406736(13)60595-4/abstract

24. Gavina C. Doença renal crónica e doença aterosclerótica cardiovascular: o risco cardiorrenal. Rev Port Cardiol. 2010; (29): 19-25. [acesso em 7 julho 2014]. Disponível em: http://www.spc.pt/dl/rpc/ artigos/1208.pdf \#page $=20$

25. Potter PSA, Perry, AG. Fundamentos de enfermagem. Rio de Janeiro: Elsevier; 2009.

26. Abensur H. Deficiência de ferro na doença renal crônica. Rev Bras Hematol Hemoter. [Internet] 2010; 32 Suppl 2 [acesso em 8 julho 2014]. Disponível em: http://www.scielo.br/pdf/rbhh/v32s2/aop47010.pdf

27. Canziani MEF, Bastos MG, Bregman R, Filho RP, Tomiyama C, Draibe SA, et al. Deficiência de ferro e anemia na doença renal crônica. J Bras Nefrol. 2006; 28(2): 86-90 [acesso em 8 julho 2014]. Disponível em: http://goo.gl/XOiAEg

28. Silva GD, Acúrcio FA, Cherchiglia ML, Guerra AA 
Jr, Andrade EIG. Medicamentos excepcionais para doença renal crônica: gastos e perfil de utilização em Minas Gerais, Brasil. Cad Saúde Pública. [Internet] 2011; 27(2) [acesso em 13 julho 2014]. Disponível em: http://www.scielo.br/scielo.php?pid=S0102311X2011000200017\&script=sci_arttext

29. Bastos MG, Kirsztajn, GM. Doença renal crônica: importância do diagnóstico precoce, encaminhamento imediato e abordagem interdisciplinar estruturada para melhora do desfecho em pacientes ainda não submetidos a diálise. J Bras Nefrol. [Internet] 2011; 33(1) [acesso em 13 julho 2014]. Disponível em: http:// www.sbn.org.br/podcast/artigo2.pdf 